\title{
Entretien avec Colette Fellous au sujet de la collection « Traits et portraits »
}

\section{Brigitte Ferrato-Combe}

\section{(2) OpenEdition}

12 Journals

Édition électronique

URL : http://journals.openedition.org/recherchestravaux/374

DOI : 10.4000/recherchestravaux.374

ISSN : 1969-6434

Éditeur

UGA Éditions/Université Grenoble Alpes

\section{Édition imprimée}

Date de publication : 30 décembre 2009

Pagination : $57-66$

ISBN : 978-2-84310-159-5

ISSN : 0151-1874

\section{Référence électronique}

Brigitte Ferrato-Combe, «Entretien avec Colette Fellous au sujet de la collection « Traits et portraits » », Recherches \& Travaux [En ligne], 75 | 2009, mis en ligne le 30 juin 2011, consulté le 07 septembre 2020. URL : http://journals.openedition.org/recherchestravaux/374 ; DOI : https://doi.org/ 10.4000/recherchestravaux.374 


\section{Entretien avec Colette Fellous au sujet de la collection «Traits et portraits»}

Colette Fellous, romancière, essayiste, productrice de l'émission Carnet nomade sur France-Culture, a créé en 2004 au Mercure de France une collection, "Traits et portraits", qui se définit comme une collection d'autoportraits : "[elle] accueille et réunit écrivains, poètes, cinéastes, peintres ou créateurs de mode. Chacun s'essaie à l'exercice de l'autoportrait. Les textes sont ponctués de dessins, d'images, de tableaux ou de photos qui habitent les livres comme une autre voix en écho, formant presque un récit souterrain ${ }^{\mathrm{I}}$."

Quinze titres sont parus à ce jour : Jean-Christophe Bailly, Tuiles détachées (février 2004), Jean-Marie Gustave le Clézio, L’Africain (février 2004), Pierre Alechinsky, Des deux mains (avril 2004), J.-B. Pontalis, Le Dormeur éveillé, (octobre 2004), Christian Lacroix, Qui est là? (novembre 2004), Marie Ndiaye, Autoportrait en vert (décembre 2004), Roger Grenier, Andrélie (février 2005), Christian Bobin, Prisonnier au berceau (septembre 2005), Jan Voss, À la couleur (février 2006), Pierre Guyotat, Coma (avril 2006), Willy Ronis, Ce jour-là (novembre 2006), Richard Millet, L'Orient désert (juillet 2007), Rosetta Loy, La Première Main (décembre 2007), Denis Podalydès, Voix off (août 2008), et Catherine Cusset, New York journal d'un cycle (février 2009). Tous les volumes, brochés, de format identique (I4 x $20,5 \mathrm{~cm}$ ), sont ornés d'un même graphisme de Pierre Alechinsky, traçant les lettres du titre de la collection en blanc ou noir sur une couverture toujours de couleur vive mais chaque fois différente. La majorité des titres ont été repris dans la collection «Folio» chez Gallimard, à l'exception des ouvrages les plus richement illustrés.

I. Texte de présentation de la collection sur le site du Mercure de France. 
Invitée à participer à la journée d'étude sur "L'autoportrait fragmentaire» organisée en mai 2007 à l'université Stendhal, Colette Fellous avait présenté sa collection et retracé le parcours personnel qui l'a conduite du séminaire de Roland Barthes à la conception de ce projet. Elle a bien voulu préciser ses réponses lors d'un entretien avec Brigitte Ferrato-Combe, réalisé en décembre 2008 .

Brigitte Ferrato-Combe La collection "Traits et portraits", que vous avez créée en 2004 au Mercure de France, trouve-t-elle son origine dans votre propre travail d'écrivain?

Colette Fellous Oui, en écrivant Avenue de France, en 200I, le premier livre d'une trilogie autobiographique (que j'ai terminée à présent puisque le deuxième livre est Aujourd'hui et le troisième Plein été $)$, je me suis aperçue que j'avais eu un grand plaisir à travailler une matière qui mêlait très librement photos, images et textes. C'était la première fois que je découvrais cette façon d'avancer dans un livre en compagnie de documents iconographiques et je les voyais prendre un tout autre visage dès qu'ils étaient associés à mon récit. Ils avaient leur propre battement et donnaient du relief au texte, ils devenaient une espèce "d'arrière-pays». J'ai vécu cette expérience comme une réelle découverte et aussitôt le livre fini, j'ai eu envie de la faire partager à d'autres écrivains.

Ce qui est curieux, c'est aussi la démarche qui m’a conduite à utiliser une iconographie pour ce livre. Je savais que je ne voulais pas seulement partir de ma propre expérience d'avoir grandi en Tunisie, j'avais plutôt envie d'éclairer les liens qu'il y avait entre la Tunisie et la France tout au long d'un siècle, d'élargir donc la scène de ma mémoire. À partir de la matière même de la ville, de son architecture, des passants qui traversaient les différents quartiers, des monuments, des gares, des carrefours, des cafés. Je voulais dépasser mon seul regard et ne plus me soucier de la chronologie, je voulais me projeter à des points différents du temps, franchir la frontière de ma propre mémoire pour rencontrer celle de mes ancêtres par exemple, ou celle d'autres passants de la fin du XIx ${ }^{\mathrm{e}}$ siècle, quand l'histoire de la colonisation commençait à peine. Raconter librement l'histoire d'une ville, son histoire à la fois politique et affective, sur Ioo ans.

Il fallait donc que je trouve une forme littéraire très fluide pour raconter ça et pendant que j'écrivais, je m'entourais de documents, de cartes postales, de photos, de publicités de l'époque, d'images de cinéma aussi, qui m’inspiraient beaucoup pour passer d'un lieu à un autre, d'une époque à une autre,

2. C. Fellous, Avenue de France; Aujourd'hui; Plein Été, Gallimard, 200I ; 2005 ; 2007. 
dans la même phrase parfois. J'installais ce "décor» autour de moi, sur ma table de travail, c'était à la fois un rempart que je me fabriquais et un texte parallèle. Je ne pensais pas du tout au départ intégrer ces éléments dans mon roman, mais j'avais besoin de leur présence. Quand je changeais de maison, par exemple quand j'allais à la campagne pour travailler, $j$ 'emportais tout et reconstituais aussitôt ce paysage autour de mon texte, avant de me mettre à travailler. Je ne pouvais plus avancer sans cette présence. À un moment, j'ai compris que tous ces documents n'étaient pas extra-littéraires mais qu'ils devaient faire partie du texte, qu'ils n'étaient plus un décor mais une partie du texte lui-même. Ils n'étaient ni illustratifs ni complémentaires du récit, ils avaient leur propre voix, ils étaient le récit. À partir du moment où j'ai compris ça, j'ai demandé chez Gallimard si je pouvais insérer des photos dans mon texte et quand Teresa Cremisi et Antoine Gallimard m'ont dit oui, tout d'un coup, ça m'a donné une liberté nouvelle, non seulement dans la forme mais vraiment dans la phrase. J'avais tous les droits, ma mémoire devenait très souple, je jonglais avec une mémoire plus ancienne et grâce à la présence de ces photos et de ces images, je croyais me promener dans un film. Toute une partie cinématographique entrait en jeu, je pouvais raconter pour tous, au-delà de ma propre histoire, j'avais mis une distance entre mon corps et la grande Histoire grâce à cette présence de l'iconographie. Quand j'ai terminé le livre, j'avais le sentiment d'avoir eu accès à un trésor et l'idée de la collection est née de cette joie. Il fallait que je crée une collection qui permette à d'autres écrivains et d'autres artistes de tenter cette expérience, tel a été mon rêve. Le thème de l'autoportrait est arrivé très vite parce que je voulais que la ligne de la collection soit souple et intime, profonde, comme un travail d'archéologue pour chacun.

B. F.-C. Outre cette expérience personnelle d'écrivain, et le désir de la faire partager, la collection "Traits et portraits" trouve-t-elle une origine dans vos expériences de lectrice?

C. F. Oui, je me suis souvenue de ma passion pour la collection «Les sentiers de la création" chez Skira. J'attendais chaque nouvelle publication comme on attendait un film de Godard ou de Truffaut, les textes étaient tous très puissants et la fabrication même était exemplaire. Je me souviens des livres de Pierre Alechinsky, d'Octavio Paz, d'Yves Bonnefoy. Ceux de Roland Barthes et de Francis Ponge. L'Empire des signes et La Fabrique du pré, font partie de leurs plus beaux livres. Et puis plus tard, le Roland Barthes par lui-même a été un livre très important pour moi. 


\section{B. F.-C. Vous avez été l'étudiante de Roland Barthes?}

C. F. Oui, entre 1972 et 1976, j'ai suivi son Petit Séminaire, à l'École des hautes études. On peut dire que nous avions suivi aussi la fabrication de ce livre comme nous avions suivi Le Plaisir du texte ou Fragments du discours amoureux. C'était une chance d'être là, à ce moment-là. Mais la forme même de ce livre était une espèce de modèle, entre le roman et l'essai. Barthes nous rendait complices de ses recherches, je me souviens qu'il nous avait distribué une liste de mots pour que nous écrivions à partir d'eux, mais c'étaient ses mots à lui. Je me souviens avoir choisi "adolescence» et "Charlot", mais je ne sais plus ce que j'en ai fait, si j'ai développé quelque chose à partir d'eux ou pas. Ils figurent dans son livre. Il avait l'art d'éclairer le réel, d'ouvrir les disciplines, de nous pousser à mieux voir, à mieux lire. Et tout cela dans le plaisir de l'échange, de la conversation. Aujourd'hui, je peux dire qu'il a été mon maître. Au début, je pensais que je serais essayiste : la façon qu'avait Roland Barthes d'éclairer les textes me plaisait infiniment car elle était très personnelle, très stimulante. J'écrivais dans mes cahiers depuis l'adolescence mais je ne pensais pas écrire de romans, c'est vraiment lui qui m’a poussée à oser dire «je», à ne pas perdre le «je» dans l'essai.

Il a libéré aussi la critique en nous autorisant à croiser les disciplines, à parler autant de peinture que de musique ou de cinéma. Nous pouvions accorder des choses discordantes, utiliser nos failles, nos défauts, il s'agissait de tout transformer au bon moment et de trouver sa voix juste, c'est en tout cas ainsi que j'ai compris son enseignement. Il nous éveillait, de la même façon que François Warin, mon professeur de philosophie en Tunisie, nous avait éveillés à tout comprendre d'un coup, les sciences, les mathématiques, la géographie, l'histoire : en philosophie, tout vivait en amitié, en solidarité.

Quand j'ai créé cette collection, j'ai retrouvé ce plaisir de croiser les disciplines et j'ai voulu l'ouvrir à d'autres artistes, à des peintres, des photographes, des stylistes, des acteurs, peut-être à des cuisiniers, pourquoi pas? Chacun doit montrer ici son savoir-faire, sa manière de penser et de rêver le monde, de le construire. En même temps, c'est une collection très artisanale. Je peux rester des mois avant de demander à quelqu'un d'écrire un livre, j'aime que les projets naissent lentement, c'est un parcours qui peut ressembler à la maturation de l'écriture, je vois ce métier d'éditeur aussi comme ça. C'est la prolongation de mon travail d'écrivain, mais avec les autres, avec l'écriture des autres et même si les textes sont complètement à eux, pour moi c'est de l'écriture aussi que j'ai accomplie avec eux, ou à côté d'eux. Quand un texte est réussi, c'est une grande joie à deux. 
B. F.-C. Revenons au séminaire de Barthes. Il y a quelques semaines, Chantal Thomas a proposé à l'université de Grenoble une conférence qui sintitulait: "De l'essai au roman". Elle a évoqué son expérience du séminaire de Barthes, qu'elle avait suivi avec vous, racontant que vous aviez présenté un exposé en commun sur la danse. Ses propos rejoignaient les vôtres sur le passage de l'essai au roman, même si elle a pour sa part accompli d'abord son projet de thèse.

C. F. Oui, c'est pareil. Il est vrai que j'ai laissé tomber ma thèse pour écrire mon premier roman... Il y avait une grande effervescence dans ce séminaire, nous étions tous assez survoltés, Barthes nous donnait le goût de vivre, de penser, de s'aimer, de rire, d'être frivole et grave en même temps, quel enseignement! Il nous donnait le goût des conversations infinies, décalées, drôles. Avec Chantal Thomas, nous passions des heures à parler et à rire. Il éveillait la langue personnelle de chacun et en bon maitre, il entretenait avec chacun d'entre nous une relation inédite, si bien qu'un tissu merveilleux de séduction nous enveloppait et que nous étions tous amoureux les uns des autres. Et Barthes, qui souriait mystérieusement face à nous... Mais son analyse des textes était incomparable. C'est d'ailleurs après avoir lu $S / Z$ que j'ai eu envie d'entrer dans son séminaire parce qu'il avait trouvé là une façon singulière d'ouvrir le texte, de l'élargir, de le faire respirer, qui m'avait éblouie, encore plus que dans le Système de la mode ou les Mythologies.

\section{B. F.-C. Comment vous avait-il accueillie?}

C. F. Chantal Thomas, à la fin de son livre sur les cafés ${ }^{3}$, se souvient de sa première rencontre avec Roland Barthes, rue de Tournon, quand elle a d'abord croisé au bout du couloir un homme qu'elle avait pris pour un appariteur et qui était bien Roland Barthes, quand ils ont ensuite commencé à parler et qu'en partant elle a fait tomber une pile de livres qui était sur la table et que tous deux se sont mis à ramasser les livres, un peu timides. Cette image des livres qui tombent sans fin et de cette légère gêne, c'est vraiment Barthes. J'ai l'impression qu'à chaque fois, c'était comme une rencontre amoureuse avec lui, un moment qui s'est vraiment détaché de tous les autres et qui a fixé un engagement, un contrat, presque un lien entre étudiant et maître. Je me souviens que j'étais allée le voir un peu tard, les inscriptions étaient finies mais je voulais absolument être dans ce séminaire, je me disais que «ce serait mon bonheur». Je le lui ai expliqué, sans doute maladroitement parce qu'il m’intimidait beaucoup et que je n'avais que vingt-deux ans. On était debout dans son bureau et il m’a dit : «je suis désolé, mais c'est trop tard», de sa voix bien basse, «c'est dommage, mais je ne prends plus d'étudiants cette année, je

3. Ch. Thomas, Cafés de la mémoire, Seuil, 2008. 
voudrais créer des séminaires restreints». Et là, j’ai senti que je ne pouvais pas laisser passer ce moment, il fallait que j'insiste et j'ai dit : "c'est très important pour moi et j'ai besoin d'une présence lointaine». J'ai senti alors dans ses yeux une espèce de mouvement, il a souri et m'a dit : «alors, c'est d'accord ».

\section{B. F.-C. La séduction de la formule?}

C. F. Peut-être, oui, mais je me souviens précisément de l'urgence qu'il y a eu dans mon corps à ce moment-là. Le séminaire était ensuite merveilleux, c'était une espèce de théâtre et pour moi, des années essentielles dans ma formation. Quand j'ai commencé à éditer les premiers livres de la collection, j'ai immédiatement pensé que j'aurais adoré lui proposer d'en écrire un, s'il était vivant, même si en un certain sens il l'avait déjà écrit son autoportrait...

B. F.-C. Comment les auteurs auxquels vous vous adressez entendent-ils ce mot "autoportrait»? comment le reçoivent-ils? Est-ce quil s'agit pour eux d'une contrainte?

C. F. Les réactions sont à chaque fois différentes. Certains me disent que tous leurs livres sont déjà des autoportraits, d'autres au contraire n'ont pas envie de dire "je». Mais il n'y a pas de réelle contrainte à dire «je», c'est ce que je leur dis au départ. On peut raconter une promenade, un moment précis de sa vie, on peut évoquer un lieu, un objet, le livre peut être un seul récit ou être sous forme de fragments comme Le Dormeur éveillé de J.-B. Pontalis, chacun doit en réalité trouver son objet qui permet d'éclairer son œuvre tout entière.

\section{B. F.-C. C'est parfois le portrait d'un autre aussi...}

C. F. Oui, Roger Grenier a choisi de décrire sa mère dans Andrélie, mais il l'a vue comme si elle était un personnage de ses livres, ce qui donne un portrait très puissant d'ailleurs. Marie NDiaye a écrit une chose très mystérieuse à partir de femmes en vert, mais ce mystère lui ressemble, c'est vraiment elle, ce livre, et en cela, c'est vraiment un autoportrait. Chaque livre est un pas de côté dans l'œuvre de chacun et doit servir à l'éclairer. Cette collection est aussi un désir de lecteur. Il y a un grand plaisir à faire naître un nouveau livre d'un auteur que vous aimez, un livre insolite, légèrement différent des autres, enfin, je crois...

B. F.-C. Oui, le résultat est là, indéniablement... Pouvez-vous préciser comment vous choisissez les auteurs de la collection?

C. F. Mes choix sont plutôt intuitifs, c'est comme une palette, les auteurs sont tous très différents et n'appartiennent à aucune école, mais je crois que 
ce qui les relie, c'est qu'ils ont chacun une grande liberté et se sont construits justement en dehors de tout mouvement littéraire ou artistique. Parfois, j'aimerais demander un livre à un auteur qui m’intéresse mais je sens qu'il n’irait pas vraiment avec les autres, alors j'abandonne ce projet.

B. F.-C. Vous avez étendu la palette par rapport à ce qui était prévu, vous annonciez: "écrivain, poètes, cinéastes, peintres ou créateurs de mode", et il y a aussi un acteur, un photographe...

C. F. Oui, c'est vrai. Je tiens beaucoup à ce qu'il y ait ce mélange de genres, c'est un des aspects essentiels de la collection. Mais j'aimerais ouvrir encore la collection à des cuisiniers, des médecins, des scientifiques...

B. F.-C. Est-ce quil y a eu des refus, des auteurs qui ont refusé de le faire, ou qui n'ont pas réussi?

C. F. Le livre de Richard Millet, L'Orient désert, n'est pas le livre qu'il devait écrire. Il voulait bâtir une chose très particulière sur son amour des femmes, mais finalement il a renoncé et l'a transformé en un livre sur Beyrouth, avec seulement quelques traces de ce qu'il aurait dû écrire. Mais des refus, il n'y en a pas eu vraiment, plutôt des hésitations, des attentes, des auteurs qui n'ont pas encore fini leurs livres mais c'est la vie de l'édition, avec ses surprises, ses retards, ses rebondissements. Un livre que j'attends toujours, c'est celui d'Arnaud Desplechin, qui est pratiquement fait mais qu'il hésite à voir publier ; on ne sait jamais, il faut beaucoup de patience... D'autres sont en cours mais tant qu'ils ne sont pas terminés, je préfere ne pas les nommer.

\section{B. F.-C. Pas de regrets d'éditrice?}

C. F. Bien sûr, j'aurais aimé voir figurer au catalogue Roland Barthes, Vladimir Jankélévitch, Robert Kramer, Jean Eustache, Hervé Guibert...

B. F.-C. Dans le principe de cette collection, les "textes sont ponctués de dessins, d'images, de photos, de tableaux" qui habitent les livres comme "une autre voix en écho, formant presque un récit souterrain". À propos des images d'Avenue de France, vous disiez qu'elles sétaient constituées en récit. Comment s'effectue le choix des images, non seulement leur sélection mais aussi leur disposition par rapport au texte? Qui préside à cette répartition?

C. F. Cela dépend bien sûr de chaque livre. La maquettiste, Anne Lagarrigue, suit la collection depuis le début, et nous travaillons en étroite collaboration avec Isabelle Gallimard qui dirige le Mercure et qui supervise bien entendu toute la fabrication et la production. Par exemple, pour L'Africain de JeanMarie Le Clézio, j'ai établi moi-même le choix et la place de chaque élément et je les ai proposés ensuite à Le Clézio qui les a acceptés tel quel. Il m’avait 
donné de nombreuses photos faites par son père mais je ne voulais pas que ce soit trop illustratif par rapport au récit. Bien entendu, Jan Voss, Pierre Alechinsky, Willy Ronis étaient, eux, très près de la fabrication et c'était pour moi une chance de pouvoir suivre leur travail jusqu'au bout; nous discutions de la place de chaque élément, nous faisions des essais, nous reprenions, etc. Jean-Christophe Bailly, par exemple, était réticent au début à cette idée d'iconographie, mais il a ensuite pris beaucoup de plaisir à mettre en scène ses éléments.

B. F.-C. Dans le livre de Bailly, il y a un écho par moments très précis entre le texte et les images en regard... Il y a un rapport métaphorique entre le texte et limage.

C. F. Oui, il a préféré jouer avec l'illustratif, mais aussi avec les répétitions, les échos, $\mathrm{j}$ 'ai aimé le voir travailler ainsi. C'est une conversation entre deux récits amis mais qui ne parlent pas vraiment la même langue. J.-B. Pontalis, lui, voulait plutôt partir des images et non pas le contraire. C'était son choix et je crois que le texte qui est né de ce choix lui ressemble vraiment.

B. F.-C. D'ailleurs son texte est fragmenté, par séquences.

C. F. Oui, il m'envoyait son texte par petits chapitres, régulièrement, comme un feuilleton, sans contrainte. Chaque livre devrait être écrit dans le plaisir, ce serait d'ailleurs ça, le contrat tacite de la collection. J'avais l'impression de recevoir des bouquets de fleurs dans ma boîte aux lettres et le livre s'est fabriqué ainsi, pas à pas, fragment par fragment, comme un jeu.

\section{B. F.-C. C'est lui, ensuite, qui les a réorganisés?'}

C. F. Oui, bien sûr. Mais j'ai dû vraiment insister pour qu'il accepte de mettre la photo de lui enfant devant la mer, juste après la mort de son père, une merveilleuse photo. Il ne voulait pas apparaître directement, mais je sentais bien que c'était une photo essentielle dans le trajet du livre. Il n'a d'ailleurs, je crois, pas regretté de l'avoir mise, par la suite...

B. F.-C. Vous dites, et on le vérifie à la lecture, que les images constituent un récit parallèle, mais en même temps, leur présence introduit une certaine discontinuité. Est-ce que le caractère fragmentaire fait partie du projet?

C. F. Oui, le fragment tient de l'autoportrait, il nuance, il éclaire, il peut jouer avec des temps différents, des lieux différents. L'autoportrait est une espèce de constellation. L'exemple le plus typique pourrait être le livre de Denis Podalydès sur la voix. Le thème est défini, c'est la voix, mais tout autour bruissent des fragments, des échos, des portraits de famille, d'amis, de 
maîtres, c'est précisément le caractère fragmentaire de son livre qui signe ici l'autoportrait.

B. F.-C. Et puis il y a ce fragment quil ne développe jamais, "voix de mes frères", qui revient...

C. F. Quand le livre était en cours d'écriture, il avait laissé quelques titres en blanc, sans les développer encore, mais quand j'ai vu "Voix de mes frères", je lui ai suggéré de ne jamais développer ce thème essentiel chez lui, de faire vivre ce blanc, de lui donner toute sa majesté. Je lui ai même proposé de le faire revenir, en refrain.

B. F.-C. Ça revient comme un refrain, un motif lancinant, ou comme un germe, un fragment enfoui, indicible, dont tout le reste serait le développement...

C. F. Exactement, c'est à partir de là qu'il écrit tout ce livre, il y a des choses qui doivent rester silencieuses tant elles sont fortes, ou alors il faut trouver des détours pour les approcher. C'est ce qu'il a fait dans son livre. J'écris aussi à partir d'une chose impossible à dire. Dans Plein été, par exemple, je dis en ouverture du livre que j'ai un secret, mais je ne le dévoile jamais, c'est le livre entier qui est irradié par ce secret, c'est tout ce qu'il y a autour qui compte.

B. F.-C. Un autre ouvrage de la collection paraît un peu différent, hors série presque, c'est celui de Christian Lacroix, qui semble avoir une histoire particulière.

C. F. Ce livre est très important pour moi. J'avais rencontré Christian Lacroix en préparant une émission de radio sur la mode et la première fois que je l'ai vu, dans son atelier, je me souviens qu'il portait un petit foulard indien comme on en portait en 68 , noué très près du cou sur le côté. Il m'avait parlé de Modiano, de l'amour qu'il avait pour la littérature et pour le théâtre, on a parlé ensuite de Bob Wilson, de tous les spectacles qu'on avait vus dans les années 70, de toutes les grandes premières, je le trouvais vif, passionné, extraordinaire, et dès que j'ai eu la collection, j'ai pensé à lui. Il a aimé tout de suite le projet, mais il était tellement pris qu'on a mis plusieurs années avant de se décider. La création de ce livre a été fantastique parce qu'il y avait une matière très riche, très éclatée. Des photos de famille, des tableaux, des coupures de presse, des dessins. Il crée à partir de fragments, il fait des collages, il aime les empiècements, les broderies, les tissus superposés, incrustés, dévorés, et son esprit est si rapide, il traverse avec aisance toutes les époques, il prend un détail d'un tableau, le met en écho avec une scène d'enfance ou le motif d'un imprimé, c'est vertigineux et j'ai adoré l'accompagner dans sa recherche pour fabriquer ce livre. 
B. F.-C. Le volume est très beau, d'ailleurs.

C. F. C'est un jaillissement... mais pour lui il n'y avait pas encore assez de choses, il aurait voulu que ce soit beaucoup plus étonnant... Cette expérience a été importante, je crois, pour nous tous.

B. F.-C. Il y a parfois des prolongements inattendus: je pense au texte de Christian Bobin qui dans Prisonnier au berceau introduit des photos d'Emily Dickinson et publie l'année d'après une biographie d'Emily Dickinson, La Dame blanche, dans la collection "L'un et l'autre» dirigée par J.-B. Pontalis, une collection sans image mais qui entretient de nombreux liens avec la vôtre.

C. F. Oui, c'est vrai, les deux livres sont complémentaires. La Dame blanche est né de ce travail sur l'autoportrait, mais je crois qu'il avait l'intention d'écrire sur Emily Dickinson depuis longtemps.

B. F.-C. Ce qui est très troublant, c'est qu'il y a presque autant de photos d'Emily Dickinson que de lui et même à des endroits où on attendrait une photo de lui, par exemple le seul portrait qu'il y a, c'est un portrait d'elle et non pas de lui, le berceau qui motive le titre, c'est celui d'Emily ${ }^{4} . .$.

C. F. Un autoportrait est parfois l'écho d'une autre figure, plus secrète en soi, un double avec lequel on joue. On se cache, on disparaît, on revient. Je crois que Christian Bobin a vraiment ressenti cela en écrivant Prisonnier au berceau. Cette proximité avec Dickinson, le thème de l'enfermement, de la maison. Choisir d'ailleurs le berceau et la chambre de Dickinson dans l'iconographie montre bien ce parallèle qu'il a voulu souligner. L'image de l'autre aide à mettre de la lumière sur sa propre histoire.

4. Sur ce croisement des images et ces échos entre les deux textes de Chr. Bobin, voir : B. Ferrato-Combe, "La maison natale, berceau de l'écriture : Christian Bobin entre autoportrait et portrait d'Emily Dickinson", actes du colloque de Montréal, Nouvelles Écritures biographiques, octobre 2008, à paraître. 